\title{
The Effectiveness and Value of Siponimod for Secondary Progressive Multiple Sclerosis
}

\section{A Summary from the Institute for Clinical and Economic Review's Midwest Comparative Effectiveness Public Advisory Council}

\author{
Patricia G. Synnott, MS, MALD; Lisa M. Bloudek, PharmD, MS; Ravi Sharaf, MD, MS; \\ Josh J. Carlson, PhD, MPH; and Steven D. Pearson, MD, MSc
}

$\mathrm{M}$ ultiple sclerosis (MS) is an immune-mediated disease of the central nervous system. Relapsing-remitting MS (RRMS) is the most common disease course and is characterized by intermittent periods of worsening neurologic symptoms ("relapses") followed by partial or complete recovery. Incomplete recovery from relapses may contribute to progression of disability. Over time, RRMS may transition to secondary progressive MS (SPMS), wherein disability progression occurs in the absence of, or independent of, relapses.

Siponimod (Mayzent) is a sphingosine-1-phosphate (S1P) receptor modulator approved for relapsing forms of MS, which has a similar mechanism of action to fingolimod. Although there are many disease-modifying therapies (DMTs) approved for patients with RRMS and patients with SPMS who still experience relapses ("active SPMS"), therapies labeled for the treatment of nonactive SPMS are lacking. The Institute for Clinical and Economic Review (ICER) reviewed the clinical effectiveness and cost-effectiveness of siponimod for patients with active and nonactive SPMS, which represents the population where it has primarily been studied.

\section{Summary of Findings}

\section{Clinical Effectiveness}

We evaluated the evidence comparing the clinical effectiveness of siponimod to best supportive care, as estimated by the placebo arm of the pivotal trial. The primary source of evidence was the phase 3, randomized placebo-controlled EXPAND trial in patients with active and nonactive SPMS. ${ }^{1}$ In this trial, siponimod reduced the risk of 3-month confirmed disability progression, as measured by the Expanded Disability Status Scale (EDSS; hazard ratio $[\mathrm{HR}]=0.79 ; 95 \%$ confidence interval $[C I]=0.65-0.95) .{ }^{1}$ Siponimod also reduced the risk of relapse $(\mathrm{HR}=0.54 ; 95 \% \mathrm{CI}=0.41-0.70)$ and decreased measures of inflammatory disease activity on magnetic resonance imaging (MRI), including the number of gadolinium-enhancing lesions on Tl-weighted scans and number of new or enlarging lesions on T2-weighted images. Significant benefits were not observed for other mobility-related measures, including the timed 25-foot walk test and the 12-point Multiple Sclerosis

J Manag Care Spec Pharm. 2020;26(3):236-39

Copyright $\odot 2020$, Academy of Managed Care Pharmacy. All rights reserved.
Walking Scale. Full details from ICER's review are available in the final evidence report on the ICER website (https://icerreview.org/wp-content/uploads/2018/10/ICER_MS_Final_ Evidence_Report_062019.pdf).

The most frequently reported adverse events in the EXPAND trial were headache, nasopharyngitis, urinary tract infection, falls, and hypertension. Bradychardia at treatment initiation, hypertension, lymphopenia, and macular edema have been associated with S1P-receptor modulation, although these events were relatively uncommon in the EXPAND trial. Data from an ongoing 7-year open-label extension of EXPAND may provide further evidence on the long-term safety of siponimod. An integrated analysis of safety data from studies of fingolimod, another S1P receptor modulator, with up to 10 years of followup did not identify any unexpected or new safety signals. ${ }^{2}$

\section{Limitations of the Clinical Evidence}

A central issue raised by any treatment for SPMS is whether it functions by reducing relapses (and thus is effective mainly for active disease) or whether it also is able to reduce progression in the absence of relapses (and thus has efficacy in nonactive disease). In EXPAND, siponimod trended towards, but did not confer, a statistically significant improvement in confirmed disability progression for subgroups defined by the absence of gadolinium-enhancing lesions or absence of relapses in the previous 2 years. ${ }^{1}$ The U.S. Food and Drug Administration (FDA) explored this question further by conducting additional analyses in subgroups with nonactive disease (e.g., patients who did not relapse in the 2 years previous to or during the study) and concluded that results from these "analyses support the hypothesis that the delay in 3-month [confirmed disability progression] is more clearly related to the anti-inflammatory effect of siponimod (yielding a significant treatment effect on the relapsing or active aspect of the disease) than to an effect on the poorly understood 'degenerative' process felt to [dominate] the pathophysiology of SPMS." Siponimod was ultimately approved by the FDA for relapsing forms of MS, which include active SPMS but not nonactive SPMS.

\section{Long-Term Cost-Effectiveness}

We also evaluated the cost-effectiveness of siponimod versus best supportive care in the overall SPMS population (active and 
TABLE 1 Health Care Sector Cost-Effectiveness Results for Siponimod Compared with Best Supportive Care ${ }^{1}$

\begin{tabular}{|c|c|c|c|c|c|}
\hline Population & Incremental Cost, \$ & Incremental QALYs & $\begin{array}{c}\text { Cost per Additional } \\
\text { Life Year, \$ }\end{array}$ & $\begin{array}{l}\text { Cost per Life Year of } \\
\text { Ambulation, \$ }\end{array}$ & $\begin{array}{c}\text { Cost per } \\
\text { Additional QALY, \$ }\end{array}$ \\
\hline Overall SPMS population & 865,000 & 0.75 & $3,760,000$ & $1,220,000$ & $1,150,000$ \\
\hline Active SPMS population & 407,000 & 0.94 & $1,565,024$ & 329,000 & 433,000 \\
\hline
\end{tabular}

Note: Results rounded to nearest 10 thousand (overall), thousand (active). Best supportive care estimated by placebo arm of EXPAND trial. ${ }^{1}$

QALY=quality-adjusted life-year; SPMS = secondary progressive multiple sclerosis.

nonactive disease) and in the subpopulation with active SPMS. This latter population was modeled to more closely match the FDA decision to only approve siponimod for use in active forms of MS.

We developed a Markov model that consisted of 9 health states based on EDSS score and death, with a cycle length of 1 year. In the active SPMS analysis, a stopping rule was introduced based on the assumption that patients would use siponimod for the approved use in active disease and discontinue when the disease transitions to nonactive SPMS. Discontinuation was assumed to occur when patients reached an EDSS score of 7, corresponding to a point at which the average number of relapses per year begins to decline.

The model estimated confirmed disability progression, patient survival, time spent in ambulation, quality-adjusted survival, and health care costs over a lifetime time horizon. The base-case analysis used a U.S. health care system perspective with a 3\% discount rate for costs and health outcomes. The price of siponimod used in the model was based on the wholesale acquisition cost (WAC) of $\$ 88,561$ per year. ${ }^{4}$ Full details on ICER's cost-effectiveness analysis, including sensitivity and scenario analyses, are available in the final evidence report on the ICER website.

Results from our model showed that at its initial WAC price siponimod had incremental cost-effectiveness ratios of over $\$ 1,000,000$ per quality-adjusted life-year (QALY) for the overall SPMS population and $\$ 433,000$ per QALY for patients with active SPMS (Table 1). In a probabilistic sensitivity analysis, $0 \%$ of model iterations resulted in an additional cost-per-QALY result below the threshold of $\$ 150,000$ per QALY gained in either population modeled. We did not calculate value-based price benchmarks for siponimod because we did not evaluate siponimod in its full FDA-approved indication for all forms of relapsing MS.

\section{Limitations of the Cost-Effectiveness Model}

Data limitations precluded us from modeling siponimod against a more relevant comparator therapy, particularly in the active SPMS subpopulation where alternative DMTs are available. In addition, annual relapse rates by EDSS health state are known for SPMS but unavailable specifically for active SPMS. It is also uncertain whether the cost or disutility for a relapse in SPMS differs from that of a relapse in RRMS.

\section{Policy Discussion}

The Midwest Comparative Effectiveness Public Advisory Council (CEPAC; https://icer-review.org/programs/midwestcepac/) is one of the independent appraisal committees convened by ICER to engage in the public deliberation of the evidence on clinical effectiveness and cost-effectiveness of health care interventions. CEPAC is composed of medical evidence experts and leaders in patient engagement and advocacy. Their deliberation includes input from clinical experts and patient representatives specific to the condition under review, as well as formal comment from manufacturers and the public. A policy roundtable concludes each meeting during which representatives from insurers and manufacturers join with clinical experts and patient representatives to discuss how best to apply the findings of the evidence to clinical practice, insurance coverage, and pricing negotiations.

The ICER report on siponimod was the subject of a CEPAC meeting in May 2019. Following discussion, the CEPAC panel members voted 15-2 that the evidence was adequate to demonstrate that the net health benefit provided by siponimod is superior to that provided by best supportive care in active SPMS. For patients with nonactive SPMS, the panel unanimously voted that there was not adequate evidence to demonstrate that siponimod provided a superior net health benefit to that of best supportive care.

The CEPAC panel also voted on "other potential benefits" and "contextual considerations" of siponimod as part of a process intended to signal to policymakers whether there are important considerations when making judgments about long-term value for money that are not adequately captured in the analyses of clinical effectiveness and cost-effectiveness. The results of these votes are shown in Table 2 and highlight several factors that the CEPAC panel felt were important for judgments of value.

The culminating vote of the CEPAC panel, intended to integrate the elements of the value assessment framework, is normally on the "long-term value for money" of siponimod. As described in ICER's 2017-2019 value assessment framework, questions on long-term value for money are subject to a value vote when incremental cost-effectiveness ratios for the interventions of interest are between $\$ 50,000$ and $\$ 175,000$ per QALY in the primary base-case analysis. The base-case estimates of the cost per QALY for siponimod exceeded the higher end of this range; therefore, the treatment was deemed "low long-term value for money" without a vote. 


\section{TABLE 2 Potential Other Benefits, Disadvantages, and Contextual Considerations}

For patients with SPMS, does siponimod offer

1 or more of the following potential "other benefits or disadvantages" versus best supportive care not adequately captured in the clinical trial data or base-case cost-effectiveness model results?

This intervention will significantly reduce caregiver or broader family burden.

This intervention offers a novel mechanism of action or approach that will allow successful treatment of many patients for whom other available treatments have failed. This intervention will have a significant effect on improving patients' ability to return to work and/or their overall productivity.

There are other important benefits or disadvantages that should have an important role in judgments of the value of this intervention.
For patients with SPMS, are any of the following contextual considerations important in assessing siponimod's long-term value for money versus best supportive care?

Votes

6/17 $\quad$ This intervention is intended for the care of individuals with a condition of particularly high severity in terms of effect on length of life and/or quality of life.

0/17 $\quad$ This intervention is intended for the care of individuals with a condition that represents a particularly high lifetime burden of illness

\begin{tabular}{l|l}
$1 / 17$ & This intervention is the first to offer any improvement for
\end{tabular} patients with this condition.

$4 / 17$

There is significant uncertainty about the long-term risk of serious side effects of this intervention.

There is significant uncertainty about the magnitude or durability of the long-term benefits of this intervention.

There are additional contextual considerations that should have an important role in judgments of the value of this intervention
The policy roundtable discussion explored how best to translate the evidence and broader perspectives discussed into clinical practice and into pricing and insurance coverage policies. The full set of policy recommendations can be found in the final evidence report; however, the key policy recommendations are as follows:

- Evidence and clinical testimony suggested that siponimod does not have a unique role in therapy for any phenotype of MS, including active SPMS.

- Payers should offer preferential formulary status to highly effective DMTs with favorable safety profiles for patients early in their disease course (i.e., RRMS). For many patients, the evidence is not adequate to determine which DMT would be superior as a first option; therefore, it is reasonable for payers to consider step therapy as a mechanism to achieve lower costs without harming patients.

- Payers may wish to consider granting preferential formulary status to fingolimod when its generic formulation comes to the market.

\section{Conclusions}

Compared with best supportive care (i.e., placebo), siponimod significantly reduced the risk of EDSS-defined disability progression and decreased inflammatory disease activity, as measured by MRI outcomes and relapses in the general SPMS population. In subgroup analyses of patients with evidence of inflammatory disease activity, the reduction in the risk of 3-month confirmed disability progression was at least as good as in the overall population and perhaps better. Siponimod did not show a significant effect on other outcomes related to progression and ambulation. The degree to which siponimod delays progression independent of its effect on relapse activity (nonactive SPMS) remains uncertain.

\section{Authors}

PATRICIA G. SYNNOTT, MS, MALD, and STEVEN D. PEARSON, MD, MSc, Institute for Clinical and Economic Review, Boston, Massassachusetts. LISA M. BLOUDEK, PharmD, MS, and JOSH J. CARLSON, PhD, MPH, The Comparative Health Outcomes, Policy and Economics (CHOICE) Institute, Department of Pharmacy, University of Washington, Seattle. RAVI SHARAF, MD, MS, Weill Cornell Medicine, New York, New York.

AUTHOR CORRESPONDENCE: Patricia G. Synnott, MS, MALD, Institute for Clinical and Economic Review, 2 Liberty Square, Ninth Fl., Boston, MA 02109. E-mail: psynnott@icer-review.org.

\section{DISCLOSURES}

Funding for this summary was contributed by Arnold Ventures, Commonwealth Fund, California Health Care Foundation, National Institute for Health Care Management (NIHCM), New England States Consortium Systems Organization, Blue Cross Blue Shield of Massachusetts, Harvard Pilgrim Health Care, Kaiser Foundation Health Plan, and Partners HealthCare to the Institute for Clinical and Economic Review (ICER), an independent organization that evaluates the evidence on the value of health care interventions. ICER's annual policy summit is supported by dues from Aetna, America's Health Insurance Plans, Anthem, Allergan, Alnylam, AstraZeneca, Biogen, Blue Shield of CA, Cambia Health Services, CVS, Editas, Express Scripts, Genentech/Roche, GlaxoSmithKline, Harvard Pilgrim, Health 
Care Service Corporation, Health Partners, Johnson \& Johnson (Janssen), Kaiser Permanente, LEO Pharma, Mallinckrodt, Merck, Novartis, National Pharmaceutical Council, Premera, Prime Therapeutics, Regeneron, Sanofi, Spark Therapeutics, and United Healthcare.

Synnott and Pearson are employed by ICER. Bloudek and Carlson report a research agreement between the University of Washington and ICER; Bloudek reports consulting fees from Allergan, Seattle Genetics, Dermira, Sunovion, TerSera Therapeutics, Cook Regentech, and Mallinckrodt Pharmaceuticals; and Carlson reports personal fees from Bayer, unrelated to this report. Sharaf reports consulting fees from ICER.

\section{ACKNOWLEDGMENTS}

The authors thank Ellie Adair, MPA, Serina Herron-Smith, and Noemi Fluetsch, MPH, for their contributions to ICER's Midwest CEPAC report on siponimod for secondary progressive multiple sclerosis.

\section{REFERENCES}

1. Kappos L, Bar-Or A, Cree BAC, et al. Siponimod versus placebo in s econdary progressive multiple sclerosis (EXPAND): a double-blind, randomised, phase 3 study. Lancet. 2018;391(10127):1263-73.

2. Kappos L, Cohen J, Collins W, et al. Fingolimod in relapsing multiple sclerosis: an integrated analysis of safety findings. Mult Scler Relat Disord. 2014;3(4):494-504.

3. U.S. Food and Drug Administration, Center for Drug Evaluation and Research. Application Number: 209884Origls000. Clinical review. Mayzent (siponimod). 2018. Available at: https://www.accessdata.fda.gov/drugsatfda docs/nda/2019/209884Origls000MedR.pdf. Accessed January 27, 2020.

4. IBM Watson Health. IBM Micromedex RED BOOK Online. [Database]. Retrieved April 18, 2019. Available at: https://www.ibm.com/products/ micromedex-red-book. Accessed January 27, 2020.

\section{Importance of Patient-Centered Outcomes When Evaluating Treatments for Secondary Progressive Multiple Sclerosis \\ Patty Taddei-Allen, PharmD, MBA, BCACP, BCGP}

\section{COMMENTARY}

I have now moved out of the heavily funded relapsing-remitting research category to the little known, least explored category of SPMS [secondary progressive multiple sclerosis]... I may have a rough road ahead." These are powerful words from a multiple sclerosis (MS) patient obtained as a comment through the MS Coalition Patient Survey. ${ }^{1}$

In March 2019, the U.S. Food and Drug Administration (FDA) approved the use of siponimod in patients with relapsing-remitting multiple sclerosis (RRMS) and active SPMS. The manufacturer sought to receive approval for use of siponimod in nonactive SPMS, but the FDA did not agree with the subset analysis of the clinical trial data submitted. The FDA also clarified that active SPMS is a subset of RRMS and all currently approved medications for use in RRMS is also indicated to treat active SPMS. ${ }^{2,3}$

One of the major determinants by the FDA to grant approval only for the active SPMS indication was due to the subgroup analyses performed regarding patients who had the presence or absence of relapses in the 2 years before the study began. The FDA determined that, while efficacy was demonstrated against placebo in the blended SPMS patient population, this effect was

J Manag Care Spec Pharm. 2020;26(3):239-41

Copyright $\odot 2020$, Academy of Managed Care Pharmacy. All rights reserved. driven by active SPMS patients due to the effect on the inflammatory aspects of MS (e.g., relapses, MRI activity) versus those with nonactive SPMS. ${ }^{3}$

In May 2019, the Institute for Clinical and Economic Review (ICER) published its final evidence report on the clinical and economic review of the use of siponimod in active and nonactive SPMS. ${ }^{4}$ ICER determined that there is a small net benefit in patients with active SPMS when compared with the best supportive care. For patients with nonactive disease compared with best supportive care, or when looking at all patients with SPMS compared with other disease-modifying therapies, there is insufficient evidence for ICER to determine an evidence rating. The economic analysis portion of the ICER report determined that siponimod exceeds commonly accepted willingness-to-pay thresholds of $\$ 50,000-\$ 150,000$ per quality-adjusted life-years gained, when compared with best supportive care in active, nonactive, and overall SPMS populations.

The ICER report listed many sources of uncertainty, most importantly the challenge in clinically differentiating between RRMS and SPMS. In addition, it listed lack of evidence, both in long-term safety and effectiveness of the data, along with lack of patient-reported and patient-centered outcomes. The clinical trials for siponimod did not evaluate outcomes of interest to patients, such as improvement in MS symptoms, caregiver burden, mental health, and overall quality of life. 